Old Dominion University

ODU Digital Commons

Bioelectrics Publications

Frank Reidy Research Center for Bioelectrics

2011

\title{
Nanosecond Pulsed Electric Field Induced Cytoskeleton, Nuclear Membrane and Telomere Damage Adversely Impact Cell Survival
}

Michael W. Stacey

Old Dominion University, mstacey@odu.edu

P. Fox

S. Buescher

Juergen F. Kolb

Old Dominion University

Follow this and additional works at: https://digitalcommons.odu.edu/bioelectrics_pubs

Part of the Biomedical Engineering and Bioengineering Commons, Cell and Developmental Biology Commons, and the Nanotechnology Commons

\section{Repository Citation}

Stacey, Michael W.; Fox, P.; Buescher, S.; and Kolb, Juergen F., "Nanosecond Pulsed Electric Field Induced Cytoskeleton, Nuclear Membrane and Telomere Damage Adversely Impact Cell Survival" (2011). Bioelectrics Publications. 71.

https://digitalcommons.odu.edu/bioelectrics_pubs/71

This Article is brought to you for free and open access by the Frank Reidy Research Center for Bioelectrics at ODU Digital Commons. It has been accepted for inclusion in Bioelectrics Publications by an authorized administrator of ODU Digital Commons. For more information, please contact digitalcommons@odu.edu. 


\title{
Nanosecond pulsed electric field induced cytoskeleton, nuclear membrane and telomere damage adversely impact cell survival.
}

\author{
Stacey M, ${ }^{1,2}$ Fox $\mathrm{P},{ }^{2}$ Buescher $\mathrm{S},{ }^{2}$ Kolb J. ${ }^{1}$ \\ ${ }^{1}$ Frank Reidy Research Center for Bioelectrics, Old Dominion University, Norfolk, VA \\ USA \\ ${ }^{2}$ Department of Pediatrics, Eastern Virginia Medical School, Norfolk, VA USA
}

Corresponding author: Dr. Michael Stacey, Center for Bioelectrics, 4211 Monarch Way, Suite 300, Norfolk, VA 23508 USA

Email: mstacey@odu.edu

Telephone: 0017576832245

Fax: 7574511010

\begin{abstract}
We investigated the effects of nanosecond pulsed electric fields (nsPEF) on three human cell lines and demonstrated cell shrinkage, breakdown of the cytoskeleton, nuclear membrane and chromosomal telomere damage. There was a differential response between cell types coinciding with cell survival. Jurkat cells showed cytoskeleton, nuclear membrane and telomere damage that severely impacted cell survival compared to two adherent cell lines. Interestingly, disruption of the actin cytoskeleton in adherent cells prior to nsPEF exposure significantly reduced cell survival. We conclude that nsPEF applications are able to induce damage to the cytoskeleton and nuclear membrane. Telomere sequences, regions that tether and stabilize DNA to the nuclear membrane, are severely compromised as measured by a pan-telomere probe. Internal pore formation following nsPEF applications has been described as a factor in induced cell death. Here we suggest that nsPEF induced physical changes to the cell in addition to pore formation need to be considered as an alternative method of cell death. We suggest nsPEF electrochemical induced depolymerization of actin filaments may account for cytoskeleton and nuclear membrane anomalies leading to sensitization.
\end{abstract}

Keywords. Nanosecond pulsed electric fields, cytoskeleton, nucleus, nuclear membrane, telomeres, cell survival. 


\section{Introduction}

Nanosecond pulsed electric fields (nsPEF) exposures are accomplished with ultra short duration (nanosecond), high intensity (kilovolt/centimeter) pulsed electric fields. Despite the field strength the energy that is delivered in a single pulse is only in the order of several hundred millijoules per cubic centimeter at most. For pulses longer than a few hundred nanoseconds (typical charging times for cells), the charge build-up at the membrane will eventually shield the inside of the cell $[1,2,3]$. With pulse durations that are short compared to the charging time of the cell membrane, the field penetrates throughout the cell, interacting with internal structures, creating distinct effects $[1,2]$. Evidence for internal cellular effects with nsPEF parameters are well documented for membrane bound lysosomes and mitochondria, but less so for the nucleus and cytoskeleton. Morphological changes and nuclear shrinkage in tumors have been demonstrated by hematoxylin and eosin stain and transmission electron microscopy [4]. In other studies, nsPEFs induced DNA damage that was most likely a direct result of the electric fields, affected cell survival [5,6]. The large surface area, along with the high negative electrostatic charge density of all cytoskeleton filament proteins presents a strong potential for interaction with electric fields if they can reach into the cell.

The cytoskeleton is an extensive filamentous network that modulates the mechanical properties of a cell, and is also essential for functions such as locomotion and cytokinesis, as well as forming a continuous dynamic connection between cellular structures. It presents an enormous surface area on which proteins, RNA, mitochondria, and other cytoplasmic components can dock. Chemical disruption of the cytoskeleton network and inhibition of tubulin and actin polymerization has been shown to induce apoptosis [7]. Indeed, the importance of tubulin directed drugs in the induction of cell death in the treatment of cancer is well documented $[8,9]$.

The nuclear matrix is composed of many interacting structural proteins that provide a framework for chromatin organization, chromatin remodeling, DNA replication, and RNA transcription $[10,11]$. Nuclear DNA is organized into chromatin loop domains, and nuclear matrix proteins anchor the base of these loops, in a cell type specific manner that allows access to the DNA by transcription factors [12]. The association of DNA to the nuclear matrix has been exploited in cancer treatment, for example, the DNA/matrix cross-linking effects of cisplatin, which affects nuclear metabolism, spatial organization of chromatin, and depolymerization of DNA bound actin filaments, leading to cell death [13]. The nuclear cytoskeleton stabilizes the nuclear membrane and importantly acts as a bridge between the nucleus and the cytoplasm.

In this study, our objective was to describe nsPEF induced structural changes occurring at cytoskeleton and nuclear envelope and relate these findings to cell survival. We demonstrate a breakdown of the cytoskeleton and a reduction in binding efficiency of telomere specific probes that were related to cell survival. We observed a reduction in cell survival when the actin cytoskeleton had been disrupted prior to nsPEF exposure, suggesting a protective effect of the cytoskeleton network.

\section{Methods and Materials}

\subsection{Cytoskeleton}

Jurkat cells, a suspension growing T-cell leukemia derived line, were grown in RPMI growth medium supplemented with $10 \%$ bovine serum, glutamine, penicillin and streptomycin at $37^{\circ} \mathrm{C}$ under a $5 \% \mathrm{CO}_{2}$ atmosphere until $\sim 70 \%$ confluence. 
Pulsing equipment [1] was calibrated in HBSS in a $0.1 \mathrm{~cm}$ electroporation cuvette (BioRad, Hercules, CA). Cell density was adjusted to $10^{6} / \mathrm{ml}$ and $140 \mu 1$ added to the cuvette and exposed to a single $60 \mathrm{~ns}$ pulsed electric field $(60 \mathrm{kV} / \mathrm{cm}, 1.7 \mathrm{~J} / \mathrm{cc})$. Controls were sham exposed.

The adherent cell lines HeLa and SV40 Normal fibroblasts were grown on polylysinecoated coverslips and exposed in situ to a single $60 \mathrm{~ns}$ pulse $(60 \mathrm{kV} / \mathrm{cm}, 1.7 \mathrm{~J} / \mathrm{cc})$. Cells were fixed at $0,1,2$, and 4 minutes after pulsing and prepared for immunocytochemistry using Oregon green 488 phalloidin, which stains F-actin filaments. Nuclei were counterstained with DAPI.

\subsection{Cell survival}

Cell survival experiments are described in detail in [6]. On day 6, viable Jurkat cells were counted using trypan blue exclusion, and adherent cells by colony forming ability. The surviving fraction was calculated as (number of cells surviving exposure/number of cells surviving non-exposure) x 100\%. Cells survival was measured and normalized to controls. Actin filaments were disrupted using $5 \mu \mathrm{g} / \mathrm{ml}$ cytochalasin B (Sigma, St. Louis, MO), for 30-40 minutes prior to nsPEF exposure and cell survival.

2.3 Fluorescence in Situ Hybridization and Immunocytochemistry

Cell lines were exposed to a single 60ns pulse in suspension. Cells were immediately removed and fixed in 3 changes of 3:1 methanol:acetic acid before applying gently to ethanol cleaned microscope slides. Hybridization to telomeres was accomplished using a FITC labeled pan-telomere probe (Vysis, Abbot Molecular, IL. USA) following the manufacturers instructions. Briefly, slides were denatured on a preheated block $\left(80^{\circ} \mathrm{C}\right.$ for $3 \mathrm{~min}$ ), transferred to a humidified chamber and hybridized in the dark for sixty minutes at $25^{\circ} \mathrm{C}$. Coverslips were removed and the slides were washed twice in formamide solution for $15 \mathrm{~min}$ (70\% formamide, $10 \mathrm{mM}$ Tris, $0.1 \% \mathrm{BSA}, \mathrm{pH}=7.0-7.5)$ followed by three washes in Tween solution ( $1 \mathrm{M}$ Tris, $0.15 \mathrm{M} \mathrm{NaCl}, 0.08 \%$ Tween, $\mathrm{pH}=7.0-7.5$ ), 5 min each. Slides were counterstained with DAPI/antifade mixture (Vectashield Burlingame, CA, USA).

To identify the nuclear membrane specific protein Lamin B1, mouse anti-human Lamin B1 and rhodamine conjugated donkey anti-mouse monoclonal antibodies were purchased from USBiological, Massachusetts. Cells were analyzed using a Zeiss Axiovert 200m epifluorescence microscope equipped with a $100 \mathrm{~W}$ mercury lamp and a $\times$ 100/1.4 N.A. oil immersion lens. Images were captured using a CCD camera and Zeiss Axiovision 4.8 imaging software.

\section{Results}

3.1 Alteration in cell morphology in nsPEF exposed HeLa cells.

Compared to mock exposed Hela cells (Here Figure 1A), within one minute of a 60ns $(60 \mathrm{kV} / \mathrm{cm})$ pulse a change in cell morphology was evident (Here Figure 1B), with ruffling of the cell membrane. By 3-4 minutes, cells had rounded and actin filaments gave a speckled appearance, indicating a breakdown of the cytoskeleton (Here Figure 1C). Many cells exposed to nsPEF dropped from the coverslip, indicating that the cytoskeleton network is no longer anchoring the cells. Preliminary data showed that when these cells were recovered and plated into tissue culture wells, they began to adhere and form colonies, suggesting the cells had survived nsPEF exposure and were viable.

3.2 Alteration in cell morphology in nsPEF exposed Jurkat cells. 
Suspension cells show an almost immediate change to the cytoskeleton compared to mock exposed cells (Here Figures 2A and 2B), with a speckled appearance of actin filaments being observed within seconds, and from which the cell does not recover. 3.3 Telomere damage.

In interphase nuclei, telomeres are bound to the nuclear membrane and therefore a potential target for damage by nsPEF-exposure. $\mathrm{nsPEF}$ exposed $(1 \mathrm{x} 60 \mathrm{kV} / \mathrm{cm})$ or sham exposed cells were gently applied to slides for FISH using a pan-telomere specific probe. As expected, unexposed cells showed telomere sequences present in the DAPI stained nucleus (Here Figure 3A). In nsPEF exposed Jurkat cells, there were no telomere signals visible. By using image enhancement (Zeiss axioscope) we were able to visualize telomere sequences distal to, but not present in, the nucleus (Here Figure 3B). Cells from an SV40 transformed normal fibroblast cell line did not show telomere damage in nsPEFexposed cells (Non-enhanced, Here Figure 3C), and have telomeres located within the main body of the nucleus. Shearing of the nuclear membrane from the nucleus was also observed in nsPEF exposed Jurkat cells (Here Figure 3D).

Telomeres were counted in 50 exposed and unexposed Jurkat cells and a significant difference noted. Sixty one signals were counted in unexposed cells (normalized to $100 \%$ ). We observed a reduction in telomere numbers after a single pulse, (7 signals, $11.5 \%$ compared to unexposed), but when the images are software enhanced telomeres become visible and numbers observed are close to unexposed (56 signals, 92\% compared to unexposed). This indicates that the probe was no longer binding to telomere sequences as efficiently as unexposed Jurkat cells, suggesting conformational changes of the target sequences. Five pulses demonstrate a reduction in telomere count ( 8 signals, $13 \%$ ), with no additional damage compared to a single pulse. These data demonstrate that nsPEF applications compromise the telomere/nuclear envelope structure.

3.4 Cell survival.

Following a single 60ns exposure Jurkat cells showed survival of $14.8+/-2.5 \%(n=4)$, HeLa cells $92.7+/-1.9 \%(n=3)$ and SV40 Normal fibroblasts $100.7+/-29 \%(n=6)$ normalized against unexposed controls. The apparent sensitivity of Jurkat cells to nsPEF induced cytoskeleton and telomere/nuclear envelope damage is indicative of mechanisms for decreased cell survival. Hypothesizing that the actin cytoskeleton has a protective role against nsPEF exposure, and that disruption of the actin cytoskeleton prior to nsPEF exposure would result in decreased cell survival; we treated HeLa cells with cytochalasin $\mathrm{B}$, an agent that prevents actin polymerization, prior to nsPEF exposure. Surviving fractions (mean $\%$ survival $+/$-standard error. $n=$ number of experiments), normalized to unexposed controls, were $96.9+/-4.8 \mathrm{n}=4$ (cytochalasin B treated only), $81.9+/-5.1 \mathrm{n}=4$ (one 60nsPEF exposure), 62.4+/-7.3 n=4 (cytochalasin B treated prior to nsPEF exposure). Significant differences (t-test) were observed for control versus nsPEF $(\mathrm{p}=0.012)$, control versus cytochalasin $\mathrm{B}+\mathrm{nsPEF}(\mathrm{p}=0.002)$ and cytochalasin $\mathrm{B}$ treated versus cytochalasin $\mathrm{B}+\mathrm{nsPEF}(\mathrm{p}=0.008)$, indicating that internal mechanical breakdown of the cytoskeleton increases the susceptibility of HeLa cells to nsPEFs.

\section{Discussion and conclusion}

Following nsPEF application, cell morphology of adherent cells changed as cell borders retreated and cells rounded due to an apparent breakdown of the cytoskeleton. The speckled appearance of the cytoskeleton in rounded cells is similar to those seen with the F-actin filament disrupting agent cytochalasin B [14, 15], suggesting 
depolymerization of actin filaments following nsPEF. This observation is in agreement with Berghöfer [16] whose report show that nsPEF applications trigger an actin response in plant cells. This group also observed a loss of nuclear shape attributed to nsPEF induced actin disruption. Although there are differences in pulse exposure (60ns, $60 \mathrm{kV} / \mathrm{cm}$ v $10 \mathrm{~ns}, 33 \mathrm{kV} / \mathrm{cm}$ ) and cell type (human cells v plant cells), the cellular response is remarkably similar. Following disruption of the cytoskeleton network with cytochalasin B prior to nsPEF exposure, we found a significant reduction in cell survival compared to unexposed cells. It appears that the internal mechanical breakdown of the cytoskeleton increases the susceptibility of adherent cells to nsPEF's, consistent with the increased susceptibility observed in suspension cells where a minimal network is present. Loss of an integral cytoskeleton may be a condition for nsPEF susceptibility.

Beaudouin [17] reported that nuclear membrane breakdown proceeds by microtubule induced tearing of the nuclear lamina. Microtubule dependent forces can deform and stretch the nuclear membrane prior to breakdown. nsPEF may cause electrochemical changes, reducing mechanical stability within the cell, leading to increased fragility of the nuclear membrane and shearing from the nucleus, as observed in Jurkat cells. Alternatively, nsPEF induced depolymerization of nuclear actin filaments may lead to nuclear membrane fragility and rupture. Conformational changes to the nuclear membrane may also account for a reduced efficiency of binding to telomere sequences that are attached to the nuclear membrane. The stability of DNA within the nucleus is achieved by attachment of the telomere ends of each chromosome to the nuclear membrane by a protein complex. A reduction in the ability of a pan-telomere probe to bind to its complementary sequences in nsPEF exposed Jurkat cells led to a reduced telomere count in interphase nuclei. Image enhancement allows the probe to become visible to near normal levels. Conformational change in the DNA/protein/nuclear membrane complex resulted in the telomere probe being unable to recognize its target sequences as efficiency as in non-pulsed cells. Interestingly, cells showing shearing of the nuclear membrane away from DAPI stained nuclei resulted in a telomere free nucleus, with telomere sequences distal to the nucleus, but still co-localized to the nuclear membrane. A reduction in telomere binding efficiency is likely a secondary consequence to changes in the nuclear membrane complex. The total number of telomere signals counted per interphase nucleus is expected to be two per chromosome (92). Our counts are lower than expected, likely due to counting hybridization signals in different planes in a 3D nucleus. Confocal microscopy using z-stacks could help resolve this. The difference in counts between exposed and unexposed nuclei is large, and we do not consider a miscount in signals to account for the observed differences.

We conclude that nsPEF applications are able to induce cytoskeleton and nuclear membrane damage and telomere sequences are severely compromised. The disruption of telomere sequences may be secondary to electrochemical changes to nuclear actin along with induced nuclear membrane fragility. Cytoskeleton actin anchors in the nuclear membrane hold proteins together in a network to stabilize this structure. These anchors maybe disrupted by pulsing, destabilizing the nuclear membrane. It appears that the minimal protection the cytoskeleton offers suspension cells allows the affects of the pulsing to be more readily felt at the nucleus, whereas in adherent cells, much of the energy is absorbed by the extensive cytoskeleton, with no nuclear effects. The telomeres are attached to the nuclear membrane. Once the nuclear membrane becomes destabilized, 
there are physical changes that occur, including the telomere/nuclear membrane binding, which reduces the efficiency of the telomere specific probe. This is not seen in adherent cells as the nuclear membrane is not destabilized. Additionally, we suggest that chemical disruption of the cytoskeleton may increase the susceptibility of cells to nsPEF applications and may be an important consideration when using pulsed electric field parameters in the destruction of tumors.

\section{Acknowledgments}

We acknowledge the support of the Jeffress Memorial Foundation and the Breeden Adams Foundation. The study sponsors had no role in study design, collection, analysis and interpretation of data; writing the report or in the decision to submit the report for publication. 


\section{References}

[1] Schoenbach KH, Beebe SJ, Buescher ES Intracellular Effect of Ultrashort Electrical Pulses. Bioelectromagnetics. 22 (2001) 440-448.

[2] Schoenbach KH, Ravindra P. Joshi, Juergen Kolb, Nianyong Chen, M. Stacey, P. Blackmore, E. Stephen Buescher and Stephen J. Beebe. Ultra short Electrical Pulses Open a New Gateway into Biological Cells. Inst. Electrical and Electronic Engineers. 92 (2004) 1122-37.

[3] Beebe SJ. Fox PM. Rec LJ. Somers K. Stark RH. Schoenbach KH. Nanosecond pulsed electric field (nsPEF) effects on cells and tissues: apoptosis induction and tumor growth inhibition. IEEE Transactions on Plasma Science. 30 (2002) 286-292.

[4] Chen X, James Swanson R, Kolb JF, Nuccitelli R, Schoenbach KH. Histopathology of normal skin and melanomas after nanosecond pulsed electric field treatment. Melanoma Res. 19 (2009) 361-371.

[5] Beebe SJ. White J. Blackmore PF. Deng Y. Somers K. Schoenbach KH. Diverse effects of nanosecond pulsed electric fields on cells and tissues. DNA Cell Biol. 22 (2003) 785-796.

[6] Stacey M. Stickley J. Fox P. Statler V. Schoenbach K. Beebe SJ. Buescher S. Differential effects in cells exposed to ultra-short, high intensity electric fields: cell survival, DNA damage, and cell cycle analysis. Mutation Research. 542 (2003) 65-75.

[7] Yamazaki Y. Tsuruga M. Zhou D. Fujita Y. Shang X. Dang Y. Kawasaki K. Oka S. Cytoskeletal disruption accelerates caspase-3 activation and alters the intracellular membrane reorganization in DNA damage-induced apoptosis. Exp Cell Res. 259 (2000) 64-78.

[8] Berrieman HK. Lind MJ. Cawkwell L. Do beta-tubulin mutations have a role in resistance to chemotherapy? Lancet Oncol. 5 (2004) 271-272.

[9] Huang YC. Guh JH. Teng CM. Induction of mitotic arrest and apoptosis by evodiamine in human leukemic T-lymphocytes. Life Sci. 75 (2004) 35-49. 
[10] Herrmann $\mathrm{H}$ and Foisner R. Intermediate filaments: novel assembly models and exciting new functions for nuclear lamins. Cell Mol. Life Sci. 60 (2003) 1607-1612.

[11] Shumaker DK. Kuczmarski ER. Goldman RD. The nucleoskeleton: lamins and actin are major players in essential nuclear functions. Current Opinions in Cell Biol. 15 (2003) 358-366.

[12] Dunn KL. Zhao H. Davie JR. The insulator binding protein CTCF associates with the nuclear matrix. Exp. Cell Research. 288 (2003) 218-223.

[13] Wozniak K and Blasiak J. Recognition of repair of DNA-cisplatin adducts. Acta Biochimica Polonica. 49 (2002) 583-596.

[14] Ujihara Y, Miyazaki H, Wada S. (2008). Morphological study of fibroblasts treated with cytochalasin D and colchicine using a confocal laser scanning microscopy. J Physiol Sci. 58 (2008) 499-506.

[15] Nagayama K and Matsumoto T. Estimation of single stress fiber stiffness in cultured aortic smooth muscle cells under relaxed and contracted states: Its relation to dynamic rearrangement of stress fibers J. Biomechanics. 43 (2010) 1443-1449.

[16] Berghöfer T, Eing C, Flickinger B, Hohenberger P, Wegner LH, Frey W, Nick P. Nanosecond electric pulses trigger actin responses in plant cells. Biochem Biophys Res Commun. 387 (2009) 590-5.

[17] Beaudouin J. Gerlich D. Daigle N. Eils R. Ellenberg J. Nuclear envelope breakdown proceeds by microtubule-induced tearing of the lamina. Cell. 108 (2002) 83-96. 


\section{Figure legends}

Figure 1. Cytoskeleton damage induced by nsPEF application. Figure 1A shows unexposed HeLa cells with an extensive actin network. One minute post pulse (1B) cells still exhibit an extensive actin network but with evidence of a ruffled outer cell membrane (arrowed). Four minutes post pulse (1C) cells have become rounded with the appearance of intensely staining actin spots (arrowed).

Figure 2. Cytoskeleton damage induced by nsPEF application. nsPEF unexposed (2A) and exposed Jurkat cells (2B). Unexposed cells showed a uniform stain over the cell, whereas exposed cells show intensely staining actin spots, suggesting nsPEF-induced breakdown of the actin network.

Figure 3. Telomere localization in Jurkat and SV40 normal fibroblast cell lines. 3A and 3C show unexposed Jurkat and SV40 transformed fibroblast cell nuclei respectively, with all telomeres located with the cell nucleus (arrowed). Figure 3B shows an enhanced image of an nsPEF-exposed Jurkat cell with telomeres (white arrow) now distal to the main body of the nucleus (black arrow). Figure 3D shows the nuclear membrane sheared away from the nucleus detected by an anti-laminB1 monoclonal antibody. 

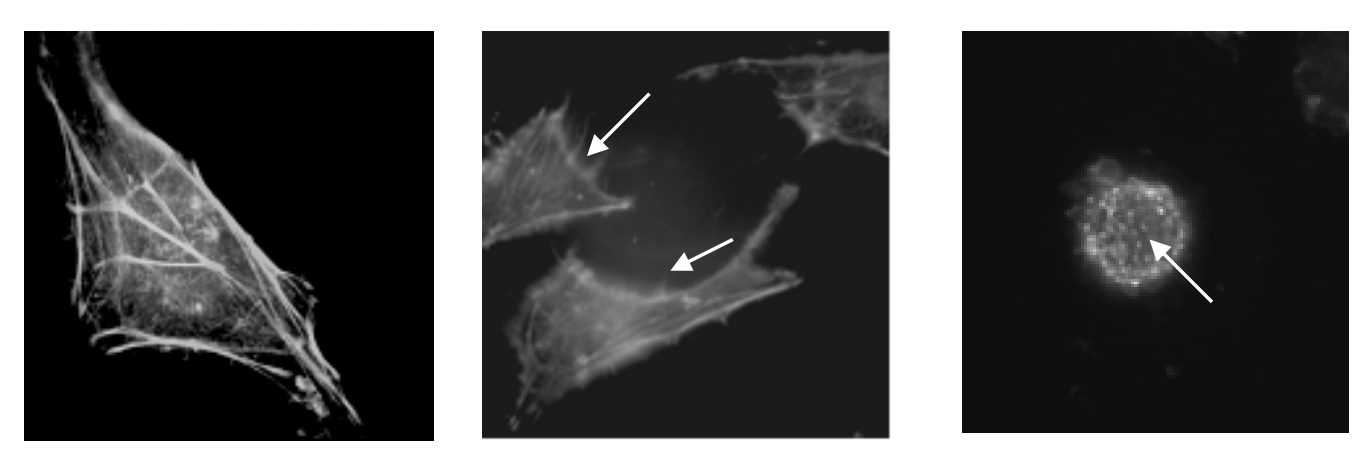

Figure 1 
Figure 3
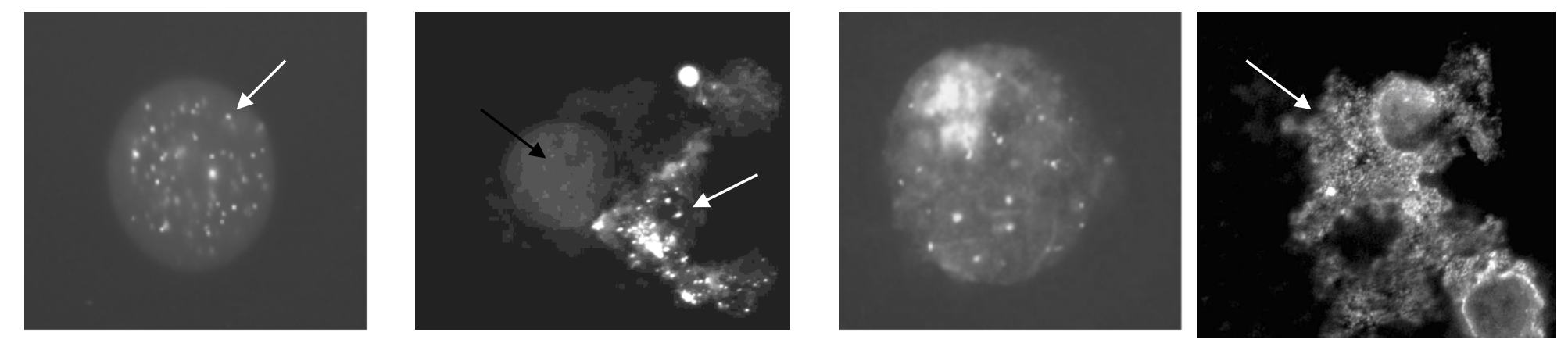


\section{Research Highlights}

1. Human cell type specific responses to nsPEFs were observed.

2. The cytoskeleton, nuclear membrane and telomeres show pulse induced damage.

3. Chromosomal telomeres are sheared away from the main body of the nucleus.

4. Disruption of the cytoskeleton prior to nsPEF exposure reduces cell survival. 